\title{
Deficiencia de glucosa-6-fosfato-deshidrogenasa (G6PD) en individuos de la ciudad de Cartagena
}

\section{Glucose-6-phosphate dehydrogenase (G6PD) deficiency in people of Cartagena city}

\author{
Angélica Quinto Casalins ${ }^{1}$, Erika Rodríguez-Cavallo ${ }^{1}$, Carlos Moneriz-Pretel ${ }^{2} \mathcal{D}$, Darío Méndez Cuadro ${ }^{3}$ \\ Facultad de Ciencias Farmacéuticas. Universidad de Cartagena.. \\ 2 Facultad de Medicina. Universidad de Cartagena. \\ ${ }^{3}$ Facultad de Ciencias Exactas y Naturales. Universidad de Cartagena. Departamento de Biología.
}

*Dirigir correspondencia a: dmendezc@unicartagena.edu.co

\begin{tabular}{l}
\hline Proceso Editorial \\
\hline Recibido: 121120 \\
Aceptado: 160321 \\
Publicado: 121121 \\
\hline
\end{tabular}

DOI 10.17081/innosa.140

(c) Copyright 2021.

Quinto $\mathrm{A}^{1}$ et al.

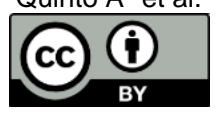

\section{RESUMEN}

Introducción: La deficiencia de glucosa-6-fosfato-deshidrogenasa (G6PD) es considerada un mecanismo genético de protección frente a las complicaciones más graves de la malaria humana. No obstante, también representa un problema de salud pública, al ser la primera causa de anemia hemolítica de origen metabólico según la OMS. Su verdadera prevalencia en la región Caribe colombiana sigue sin ser determinada con precisión. Con el objeto de obtener un panorama de esta condición en Cartagena de Indias, se realizó este estudio piloto para la identificación de individuos deficientes de G6PD en la comunidad estudiantil de la Universidad de Cartagena y sus familiares. Métodos: Estudio piloto con 434 donantes voluntarios sometidos a ensayos de tamizaje de actividad de G6PD eritrocitaria mediante la prueba de Beutler sobre papel (spot test) y confirmatorios basados en la actividad enzimática cuantitativa. Resultados: Del total de donantes, 25 resultaron sospechosos de padecer la deficiencia correspondiente, de los cuales, 20 fueron confirmados mediante la actividad enzimática cuantitativa; alcanzado una frecuencia de 4,6\% para esta afectación eritrocitaria. Conclusiones: La frecuencia de individuos deficientes de G6PD determinada en la muestra poblacional estudiada se ubica dentro los valores de prevalencia estimados para la población colombiana y ponen de manifiesto la necesidad de realizar estudios de tamizaje a mayor escala acompañados de pruebas de tamizaje molecular que permitan identificar las mutaciones con mayor penetrancia en la región Caribe colombiana.

Palabras clave: Polimorfismos eritrocitarios, glucosa-6-fosfato deshidrogenasa (G6PD), actividad enzimática, tamizaje cualitativo, deficiencia enzimática.

\begin{abstract}
Background: Glucose-6-phosphate dehydrogenase deficiency (G6PD) is considered a genetic mechanism of protection against the human severe malaria. However, it also represents a public health problem due it is the first cause of hemolytic anemia of metabolic origin, according to the WHO. Its true prevalence in the Colombian Caribbean region remains undetermined. In order to obtain an overview of this condition in Cartagena de Indias, a pilot study was carried out to identify G6PD deficient individuals in the student community of the University of Cartagena and their relatives. Methods. Identification of patients with G6PD deficiency was done by combining screening Beutler's (spot test) with kinetic confirmatory (quantitative enzyme activity) methods in the population selected. Results. 25 patients - of a total of 434 individuals - were suspected of suffering this deficiency, which accounts for a frequency of $5.7 \%$. From these, 20 were confirmed as positive through quantitative enzyme activity; resulting a frequency of $4.6 \%$ for this alteration. Conclusion: Frequency of G6PD deficient individuals in sample studied get into estimated prevalence values for the Colombian population and reinforce the need to carry out larger-scale screening studies accompanied by molecular screening tests
\end{abstract}

Keywords: Erythrocyte Polymorphism, Glucose-6-phosphate Dehydrogenase (G6PD), enzyme activity, qualitative screening, enzyme deficiency. 


\section{INTRODUCCIÓN}

La deficiencia de gluclosa-6-fos fato deshidrogenasa (G6PD) es la eritroenzimopatia más frecuente alrededor del mundo, afecta a más de 400 millones de personas y debido a su gran variabilidad genética se han identificado más de 400 variantes bioquímicas de esta enzima, las más frecuentes son la B (actividad normal), A+ (actividad normal), A- $(8 \%-20 \%$ de actividad normal), y la mediterránea $(<5 \%$ de la actividad normal) $(1,2)$.

Esta enzima reviste vital importancia porque se encuentra en todas las células del cuerpo, y su deficiencia se manifiesta marcadamente en los eritrocitos posiblemente por tener éstos una larga vida sin núcleo y organelas celulares $(3,4)$. La enzima activa consta de subunidades idénticas que forman dímeros y tetrámeros, que contiene un sitio de unión a nicotinamida-adenina dinucleotidofosfato (NADP). Este, se une a la enzima como un componente estructural y como sustrato para la reacción. La G6PD cataliza el paso de entrada de glucosa 6- fosfato (G6P) en la vía de las pentosas fosfato, específicamente en la de la hexosa monofosfato, reacción que produce oxidación de la glucosa-6-fosfato a 6-fosfogluconolactona, reduciendo NADP a NADPH; (figura1) ((2-4). Cuando se produce una deficiencia en esta enzima, se ve disminuida la acción normal de la metahemoglobina reductasa; así como, el mantenimiento de un nivel adecuado de glutatión reducido. Debido a su localización en la región terminal del brazo largo del cromosoma $\mathrm{X}$ (Xq28), los hombres son normales o deficientes, mientras que, las mujeres pueden ser normales, heterocigotas $u$ homocigotas $(1,3)$. Las mujeres heterocigotas tienen una copia del gen que sintetiza la G6PD normal y otra copia que produce la variante de la enzima; por consiguiente, dichas pacientes tienen dos poblaciones de eritrocitos, una normal y otra con deficiencia de G6PD (5).

La Organización Mundial de la Salud (OMS) estima una prevalencia de la deficiencia entre 3\% y $7 \%$ para nuestro país, sin embargo, nuestros registros acerca la prevalencia real siguen siendo incompletos. Debido a que este polimorfismo eritrocitario guarda una relación histórica con la evolución de los parásitos del género Plasmodium causantes de la malaria, y teniendo en cuenta que, la costa Caribe colombiana ha sido fuertemente influenciada por la presencia de afrodescendientes, se hace necesaria la aplicación de pruebas de tamizaje dirigidas a la identificación de individuos deficientes de G6PD. Necesidad que se ve reforzada por estudios epidemiológicos recientes que relacionan la deficiencia de G6PD con un mayor riesgo de desarrollar diabetes y enfermedades cardiovasculares $(6,7)$.

Con el fin de realizar una aproximación a la situación epidemiológica actual de este polimorfismo eritrocitario en Cartagena de Indias, se planteó este estudio piloto, donde se escogió como población representativa a la comunidad estudiantil del área de la salud de la Universidad de Cartagena y sus familiares para la realización de ensayos de tamizaje (spot test) y confirmatorios de actividad enzimática de la G6PD.

\section{MÉTODOS}

Tipo de estudio, población de referencia y selección de la muestra: Se llevó a cabo un estudio de tipo descriptivo tomando como base la población estudiantil de la universidad de Cartagena y sus familiares. Los participantes del estudio se seleccionaron de forma aleatoria simple para la identificación de los pacientes G6PD deficientes. Todos los donantes voluntarios 
suministraron su consentimiento informado en cumplimiento con las normas científicas, técnicas y administrativas vigentes para la investigación en salud establecida en el acuerdo de Helsinki, la Resolución 008430 de 1993 del Ministerio de Salud de la República de Colombia y el comité de ética de la Universidad de Cartagena. Tras lo cual se procedió a la respectiva recolección de las muestras de sangre venosa para la identificación de los polimorfismos estudiados.

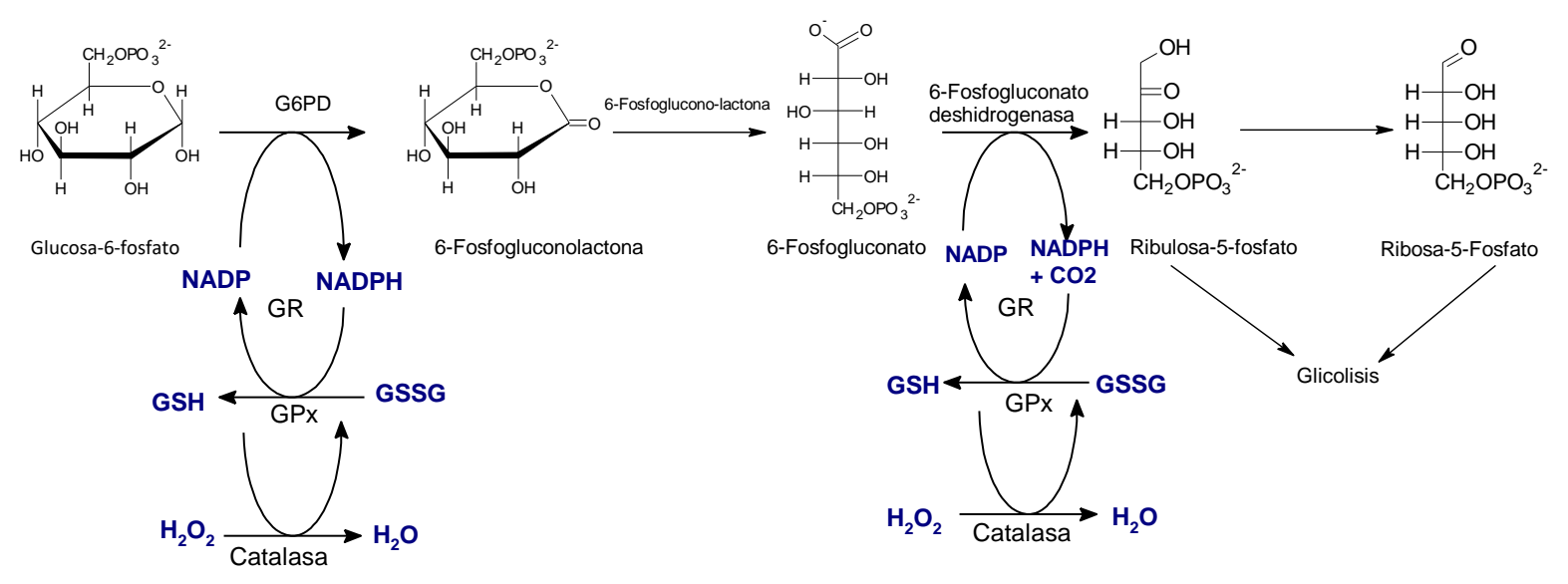

Figura 1. NADPH es producida por la acción de la G6PD y la 6-fosfogluconato deshidrogenasa en el eritrocito maduro. EI NADPH actúa como un donador de protones para la regeneración de glutatión reducido, y como un ligando para la catalasa. NADPH también actúa como un donador de protones para muchas otras reacciones enzimáticas esenciales que constituyen las defensas antioxidantes. Cat $=$ catalasa. GPX $=$ glutatión peroxidasa $. \mathrm{GR}=$ glutatión reductasa . G6PDD = deshidrogenasa de glucosa-6-fosfato. 6PGD = 6-fosfogluconato deshidrogenasa. GSH = glutatión reducido. GSSG = glutatión oxidado. Adaptado de Cappellini y col. (8)

2.1. Tamizaje cualitativo para la determinación de individuos G6PD deficientes: La identificación de este grupo de pacientes se realizó a través de diferentes métodos bioquímicos: El primero consistió en una prueba de tamizaje rápido para la identificación de deficientes potenciales mediante el uso de la técnica spot test basada en el método de Beutler modificado (9). Mediante este ensayo se determinó indirectamente la actividad de la G6PD, enzima encargada de catalizar la transformación de glucosa 6 -fosfato a gluconato-6- fosfato, con la concomitante reducción de NADP a NADPH (35). Para ello $10 \mu \mathrm{l}$ de sangre capilar, se mezclaron con $200 \mu \mathrm{l}$ del reactivo de trabajo (G-6-P $10 \mathrm{mM}$, NADP 7,5 mM, GSSG $8 \mathrm{mM}$, saponina $1 \%$ ) e incubaron en baño seco a $37^{\circ} \mathrm{C}$ por 5 y 10 minutos. Transcurridos los tiempos, $1 \mu \mathrm{L}$ de la mezcla fue sembrado en papel filtro Whatman \# 1 y se dejó secar por 20 minutos en un lugar seco y oscuro. Cumplido el tiempo, los spots fueron visualizados bajo luz UV a 365 $\mathrm{nm}$. Aquellos individuos que presentaron disminución o ausencia de fluorescencia fueron seleccionados como sospechosos de déficit de G6PD (9).

\subsection{Identificación de individuos G6PD-deficientes mediante ensayo} espectrofotométrico: La actividad enzimática de la G6PD, expresada en $\mathrm{Ul} / \mathrm{g}$ de $\mathrm{Hb}$, se determinó en muestras de sangre venosa. Para ello se emplearon tubos anticoagulados con EDTA, de donde se tomó muestra suficiente para la cuantificación de hemoglobina y la 
medición de la actividad enzimática de la G6PD. La concentración de hemoglobina se midió en sangre total utilizando un método colorimétrico basado en la formación de cianometahemoglobina (Sigma-Aldrich D5941), por reacción de la heteroproteína con el reactivo de Drabkin y lectura a $540 \mathrm{~nm}$ en un espectrofotómetro (TECAN).

En forma paralela, una parte de las muestras se sometió a centrifugación (2500 rpm por 10 minutos) para separar los eritrocitos de los demás componentes sanguíneos. $400 \mu \mathrm{l}$ de los eritrocitos obtenidos fueron lavados tres veces con $4 \mathrm{~mL}$ de $\mathrm{NaCl} 154 \mathrm{mM}$, centrifugando cada lavado en las condiciones antes descritas. Luego, $200 \mu \mathrm{l}$ de los eritrocitos lavados se suspendieron en $200 \mu \mathrm{L}$ de $\mathrm{NaCl} 154 \mathrm{mM}$, y $20 \mu \mathrm{L}$ de esta suspensión fueron lisados con 2 $\mathrm{mL}$ saponina al $0,01 \%$ mediante incubación por 10 min a temperatura ambiente. Un blanco de reactivos fue preparado y procesado de igual manera. Transcurridos los 10 minutos, se añadió $4 \mathrm{~mL}$ del reactivo de trabajo (Tris- $\mathrm{HCl} \mathrm{pH} 8,0150 \mathrm{mM}, \mathrm{MgCl}_{2}$ 14,95 mM, NADP+ 0,30 mM, G6P 1,06 mM.) a los lisados, mientras que, al blanco se le agregó $4 \mathrm{~mL} \mu \mathrm{l}$ de SS $154 \mathrm{mM}$. Luego de otros $10 \mathrm{~min}$, se registró el cambio de absorbancia por minuto durante 5 minutos a $340 \mathrm{~nm}$ para cada una de las muestras analizadas y el blanco de reactivos. La actividad de la G6PD en cada muestra se determinó de acuerdo con los lineamientos establecidos por International Committee for Standardization in Haematology: Recommended Methods for RedCell Enzyme Analysis, cuyos valores de referencia se encuentran en el intervalo de 12,1 +/$2,09 \mathrm{UI} / \mathrm{g}$ de $\mathrm{Hb}$ (9). Con el fin de obtener un valor referente en nuestra población, se determinó la actividad enzimática en un grupo de varones sanos en la prueba de spot test.

2.3 Análisis Estadístico: Para las variables cualitativas de género y de tamizaje de G6PD se utilizaron distribuciones de frecuencia y porcentuales; mientras que en el análisis de las variables cuantitativas de actividad de G6PD se usaron las medidas de tendencia central promedio y desviación estándar.

Para determinar diferencias estadísticas entre los promedios de los grupos de estudio se utilizó la prueba $t$ de Student, luego de satisfacer los supuestos de normalidad (prueba de Kolmogorov Smirnorv y Shapiro Wilks). En todos los casos el nivel de significancia fue establecido con el valor de $\mathrm{P}<0.05$.

\section{RESULTADOS}

Tamizaje cualitativo para la determinación de individuos G6PD deficientes: En cuanto a la identificación de individuos deficientes de G6PD se realizó un tamizaje aleatorio entre los estudiantes, sus familiares y trabajadores de la universidad de Cartagena (población mestiza) de este tamizaje se obtuvieron muestras de sangre de un total de 434 individuos 216 mujeres y 218 hombres y con un rango de edad de 18 a 82 años.

Las muestras obtenidas fueron analizadas mediante la técnica de spot test (figura 2), resultando 25 pacientes sospechosos de padecer la deficiencia entre ellos 13 hombres y 12 mujeres que corresponde a una frecuencia de 5,7\%. Estos individuos fueron confirmados como G6PD deficientes mediante la realización de ensayos para determinar cuantitativamente la actividad enzimática. 
A)

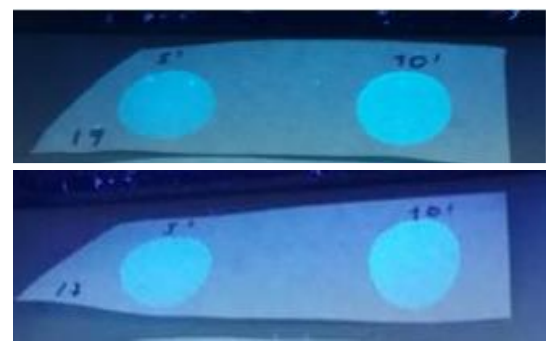

$5 \min$.

$10 \mathrm{~min}$.
B)

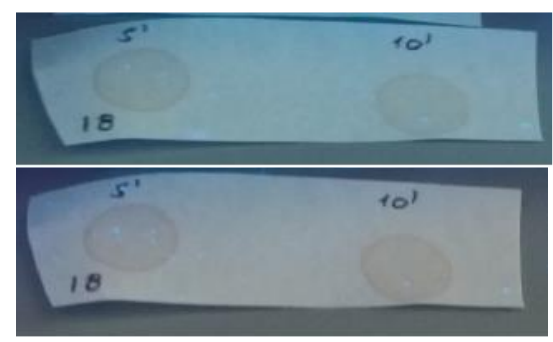

$5 \min . \quad 10 \mathrm{~min}$.

Figura 2. Resultado ensayo del ensayo de tamizaje de Beutler sobre papel filtro (spot test). La figura muestra un resultado típico obtenido en la prueba de tamizaje. A) La fluorescencia de las gotas (spots) del panel A corresponden a dos muestras de donantes con actividad normal de G6PD. La fluorescencia es debida a la formación de NADPH que se midió a los 5 y 10 minutos de ensayo, respectivamente. B) La falta de fluorescencia de las gotas en el panel B corresponde a muestras de dos donantes sospechosos de actividad deficiente de G6PD. Obsérvese la ausencia de fluorescencia a los 5 y 10 minutos, respectivamente.

Identificación de individuos G6PD deficientes mediante ensayo espectrofotométrico: Esta prueba se realizó para confirmar los pacientes sospechosos identificados por la prueba de spot test. De los 25, 20 fueron confirmados mediante esta prueba, 10 pertenecientes al género femenino y 10 del género masculino; el intervalo de edad de estos pacientes fue de 18 - 47 años y la frecuencia obtenida para esta alteración eritrocitaria fue de 4,6\%. La actividad enzimática de la G6PD del grupo de mujeres se situó en el intervalo de 4,7 a 9,3 Ul/g de Hb; mientras que el de los varones se ubicó entre 1,67 y 3,6 Ul/g de Hb. Los datos de concentración de hemogoblina y actividad enzimática de cada donante deficiente se muestran en la tabla 1, tanto para mujeres (heterocigotas) y hombres (hemicigotos). Estos datos de actividad enzimática mostraron un comportamiento normal y diferencias significativas en cuanto a género.

Tabla 1. Actividad enzimática de la G6PD en muestras de pacientes deficientes.

\begin{tabular}{|c|c|c|c|c|c|c|c|}
\hline $\mathbf{N}^{\circ}$ & $\begin{array}{c}\text { Mujeres } \\
\text { (Heterocigotas) }\end{array}$ & $\begin{array}{l}\mathrm{Hb} \\
(\mathrm{g} / \mathrm{dl})\end{array}$ & $\begin{array}{c}\text { AE } \\
\text { (UI/g de } \\
\mathrm{Hb} \text { ) }\end{array}$ & $\mathbf{N}^{\circ}$ & $\begin{array}{c}\text { Hombres. } \\
\text { (Hemicigotos) }\end{array}$ & $\begin{array}{l}\mathrm{Hb} \\
(\mathrm{g} / \mathrm{dl})\end{array}$ & $\begin{array}{c}A E \\
\text { (UI/g de } \\
H b)\end{array}$ \\
\hline 1 & $37 \mathrm{NC}$ & 12,7 & 5,9 & 11 & $35 \mathrm{DM}$ & 13,5 & 3,46 \\
\hline 2 & $341 \mathrm{MM}$ & 12,1 & 8,4 & 12 & 47JM & 13,7 & 3,39 \\
\hline 3 & 342 CM & 13,05 & 8,7 & 13 & 72 LC & 14,5 & 2,26 \\
\hline 4 & 343 MAM & 12,2 & 4,7 & 14 & $76 \mathrm{JT}$ & 13,5 & 2,18 \\
\hline 5 & 344 AM & 12,0 & 6,4 & 14 & $165 \mathrm{AD}$ & 14,7 & 1,67 \\
\hline 6 & $345 \mathrm{AP}$ & 11,8 & 8,7 & 16 & $322 \mathrm{HC}$ & 15,2 & 2,5 \\
\hline 7 & 346 CC & 12,6 & 7,4 & 17 & $349 \mathrm{CD}$ & 14,3 & 2,5 \\
\hline 8 & 348 MLA & 12,6 & 7,5 & 18 & $405 \mathrm{MM}$ & 14,3 & 1,15 \\
\hline 9 & $350 \mathrm{MP}$ & 13,1 & 8,2 & 19 & $415 \mathrm{JM}$ & 15,1 & 1,7 \\
\hline \multirow[t]{2}{*}{10} & $414 \mathrm{CG}$ & 13,3 & 9,53 & 20 & $417 \mathrm{MO}$ & 13,4 & 3,6 \\
\hline & Prom \pm DS & $12,5 \pm 0,5$ & $7,5 \pm 1,5$ & & Prom \pm DS & $14.2 \pm 0.7$ & $2,4 \pm 0,8$ \\
\hline
\end{tabular}


Aunque la actividad enzimática determinada es suficiente para identificar a los individuos como deficientes de G6PD, también se cuantificó en un grupo control de 20 varones con actividad normal de la enzima (ver material suplementario). Estos presentaron una actividad media de $21,1 \pm 5,7 \mathrm{Ul} / \mathrm{g}$ de $\mathrm{Hb}$, valor que se utilizó para determinar el porcentaje de actividad residual y la clase de deficiencia en la población estudiada.

Los datos obtenidos muestran que todas las donantes del grupo de mujeres presentan un déficit moderado deficiente de clase III con una media \pm DE de $35,7 \% \pm 7,0 \%$ de actividad enzimática residual; mientras que el promedio de los varones fue $11,6 \% \pm 3,9 \%$, con 7 de ellos en la clase III. Finalmente, tres donantes varones (165 AD, $405 \mathrm{MM}$ y $415 \mathrm{JM}$ ) presentaron valores propios de un déficit grave con menos de $10 \%$ de actividad enzimática residual. En la figura 3 muestran las diferencias en la actividad residual de individuos deficientes frente a una parte del grupo con actividad normal.

\section{DISCUSIÓN}

Los resultados obtenidos muestran que la relación entre la actividad de la G6PD, la edad y la concentración de hemoglobina no muestran diferencias estadísticamente significativas, esto es sustentado por el hecho de que solo se produce hemolisis cuando dichos individuos se exponen a agentes oxidantes (11).

Al contrastar la actividad enzimática entre los hombres y mujeres en estudio, se observan diferencias estadísticamente significativas; como se muestra en las tablas 1 y 2 . De hecho, el promedio de la actividad enzimática en mujeres $(7,5 \mathrm{Ul} / \mathrm{g}$ de $\mathrm{Hb})$ es tres veces más que lo observado en los hombres (2,4 $\mathrm{Ul} / \mathrm{g}$ de $\mathrm{Hb})$.

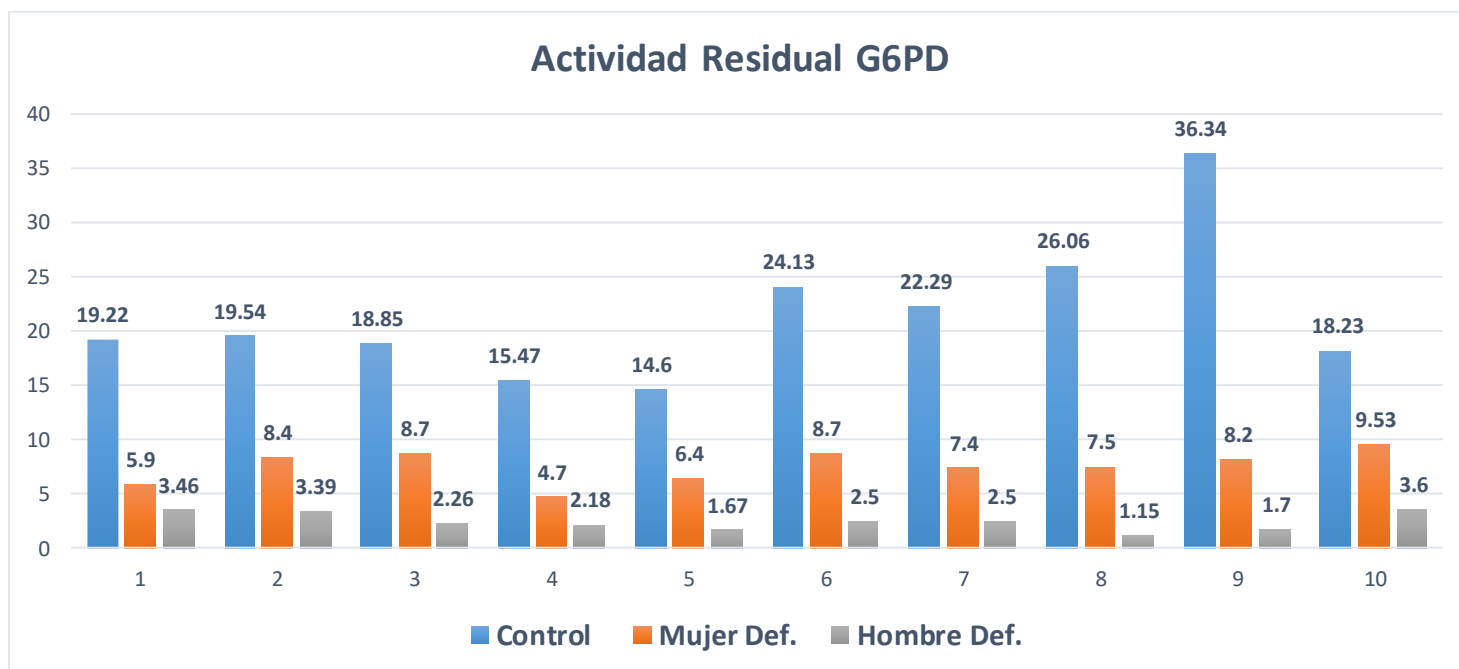

Figura 3. Actividad enzimática residual de la G6PD eritrocitaria en hombres y mujeres deficientes. Los grupos de columnas (1-10) comparan la actividad enzimática de la G6PD en una muestra de 10 controles sanos (azul), mujeres heterocigotas (anaranjado) y varones hemicigotos (gris). Todos los donantes del grupo control mostraron una actividad enzimática mayor a 12,1+/-2,09 Ul/g de $\mathrm{Hb}$. La mayor actividad residual en las mujeres se explica por su carácter heterocigoto y las diferencias fueron estadísticamente significativas entre los grupos $(P<0.05)$. 
Estas diferencias se explican por el carácter heterocigoto de las mujeres, que hace una población de sus células rojas expresen el gen deficiente y otras expresan el gen normal (12). Este mosaicismo somático es el resultado del silenciamiento genético de un alelo (lionización) (12). Aunque la cuantificación de la actividad enzimática es un método bioquímico que permite identificar mujeres con actividad baja o moderada de la G6PD, algunas de estas pueden no ser detectadas en la prueba de tamizaje empleada y por tanto la prevalencia calculada en este estudio quizás pueda ser superior.

En algunas comunidades las prevalencias alcanzan hasta 28,1\%; como es el caso de las zonas endémicas de malaria en África. Además de la ubicación geográfica, altos grados de consanguineidad, así como, el origen étnico juega un papel crucial en la distribución de esta deficiencia $(13,14)(6-9)$.

Los datos reportados para esta deficiencia en Colombia son escasos, no obstante, los que se han realizado muestran frecuencias que van desde 2.2\% en la ciudad Medellín (población blanca), $3,1 \%$ y $7 \%$ en Bogotá (población procedente de varias zonas del país), $12 \%$ en Buenaventura (principalmente población afrodescendiente) hasta 14,8\% (Turbo Antioquia) (5, 15, 16).

A nuestro saber, este estudio constituye el primer reporte en la zona Caribe colombiana y la frecuencia hallada de $4,6 \%$ es congruente con lo esperado para una zona que fue endémica de malaria, la estimación propuesta por la OMS para Colombia (3-7\%) y la importante ascendencia africana de la población.

Adicionalmente, esta frecuencia de 4,6\% de deficientes de G6PD resulta similar al 4,7\% descrito por Alvear y col para la presencia de rasgo falciforme en una muestra de 1729 recién nacidos en el periodo de enero a junio de 2019 en Cartagena de Indias (17). Frecuencias que están por encima de los 0,07 casos por 100.000 personas en 2016 y de los 0,32 casos en 2017, que reporta el Instituto Nacional de Salud para la prevalencia nacional de drepanocitosis (18).

Finalmente, nuestros sugieren que un número importante de la población Cartagenera posee algún grado de deficiencia de G6PD y que su frecuencia resulta similar al rasgo falciforme, que es otro polimorfismo protector de malaria grave. A pesar que la deficiencia de G6PD constituye un problemas de salud pública en muchos países y que recientemente ha demostrado ser un factor de riesgo cardiovascular en persona mayores de 60 años (19), su determinación no hace parte de las pruebas de tamizaje a neonatos en Colombia (20).

\section{CONCLUSIONES}

La situación actual de la deficiencia de G6PD en Cartagena de Indias y la región Caribe colombiana parece ubicarse dentro de los valores estimados por la OMS. La frecuencia calculada resultó mayor a hemoglobinopatías como la drepanocitosis que es de obligatorio tamizaje en neonatos. Por lo tanto, nuestros resultados refuerzan la necesidad de realizar de estudios subsiguientes para determinar las mutaciones del gen de la G6PD de mayor penetrancia en nuestra región y la inclusión de su diagnóstico dentro de la ley nacional de tamizaje neonatal. 
Agradecimientos: DMC y ERC agradecen a Minciencias y la Universidad de Cartagena, por el apoyo financiero al proyecto código 1170-844-67943. De igual manera a la Universidad de Cartagena por el apoyo a grupos de investigación, actas 014 de 2018 y 061 de 2019.

Declaración de intereses: Los autores declaran que no existen conflictos de interés.

\section{Contribución de los autores y subvenciones:}

AQC. Investigación, Análisis formal de datos, Redacción manuscrito original y revisión.

CMP. Investigación, Recursos materiales, Análisis formal de datos

ERC. Conceptualización, Adquisición de fondos, Administración, Redacción.

DMC. Conceptualización, Adquisición de fondos, Administración, Investigación, Recursos materiales, Redacción, Revisión, Edición.

\section{REFERENCIAS}

1. Fonseca D, Mateus H, Silva C, Contreras N, Restrepo C. Deficiencia de glucosa 6-fosfato deshidrogenasa: Aspectos generales de la eritroenzimopatía más frecuente en el mundo. Acta Medica Colombiana. 2005;30:59-64.

2. Watson JA, Leopold SJ, Simpson JA, Day NP, Dondorp AM, White NJ. Collider bias and the apparent protective effect of glucose-6-phosphate dehydrogenase deficiency on cerebral malaria. Elife. $2019 ; 8$.

3. Luzzatto L, Arese P. Favism and Glucose-6-Phosphate Dehydrogenase Deficiency. N Engl J Med. 2018;378(1):60-71.

4. Ruwende C, Hill A. Glucose-6-phosphate dehydrogenase deficiency and malaria. J Mol Med (Berl). 1998;76(8):581-8.

5. Uribe Ardila A. Deficiencia de glucosa 6-fosfato deshidrogenasa en Colombia: memorias de 22 años de tamizaje de alto riesgo. Revista Med. 2017;25(2):7-21.

6. Parsanathan R, Jain SK. Glucose-6-phosphate dehydrogenase (G6PD) deficiency is linked with cardiovascular disease. Hypertension Research. 2020;43(6):582-4.

7. Thomas JE, Kang S, Wyatt CJ, Kim FS, Mangelsdorff AD, Weigel FK. Glucose-6-Phosphate Dehydrogenase Deficiency is Associated with Cardiovascular Disease in U.S. Military Centers. Tex Heart Inst J. 2018;45(3):144-50.

8. Cappellini MD, Fiorelli G. Glucose-6-phosphate dehydrogenase deficiency. Lancet. 2008;371(9606):64-74.

9. Beutler E, Mitchell M. Special modifications of the fluorescent screening method for glucose-6phosphate dehydrogenase deficiency. Blood. 1968;32(5):816-8.

10. Beutler E, Blume KG, Kaplan JC, Löhr GW, Ramot B, Valentine WN. International Committee for Standardization in Haematology: recommended methods for red-cell enzyme analysis. British journal of haematology. 1977;35(2):331-40. 
11. Poirot E, Vittinghoff E, Ishengoma D, Alifrangis M, Carneiro I, Hashim R, et al. Risks of Hemolysis in Glucose-6-Phosphate Dehydrogenase Deficient Infants Exposed to Chlorproguanil-Dapsone, Mefloquine and Sulfadoxine-Pyrimethamine as Part of Intermittent Presumptive Treatment of Malaria in Infants. PLoS One. 2015;10(11):e0142414.

12. Chu CS, Bancone G, Nosten F, White NJ, Luzzatto L. Primaquine-induced haemolysis in females heterozygous for G6PD deficiency. Malar J. 2018;17(1):101.

13. DePina AJ, Pires CM, Andrade AJB, Dia AK, Moreira AL, Ferreira MCM, et al. The prevalence of glucose-6-phosphate dehydrogenase deficiency in the Cape Verdean population in the context of malaria elimination. PLoS One. 2020;15(3):e0229574.

14. May J, Meyer CG, Grossterlinden L, Ademowo OG, Mockenhaupt FP, Olumese PE, et al. Red cell glucose-6-phosphate dehydrogenase status and pyruvate kinase activity in a Nigerian population. Trop Med Int Health. 2000;5(2):119-23.

15. Alberto Restrepo M, Gutierrez E. The frequency of glucose-6-phosphate dehydrogenase deficiency in Colombia. American journal of human genetics. 1968;20(1):82-5.

16. Rosero MJ, Bermúdez AJ. Análisis de hemoglobinopatías en regiones afrocolombianas usando muestras de sangre seca de cordón umbilical. Acta Medica Colombiana. 2012;37:117-26.

17. Alvear CC, Barboza M, Viola M, Moneriz C, Araque LM. Pilot study of hemoglobinopathies in newborns of the Rafael Calvo maternity clinic of Cartagena, Colombia. Colombia Médica. 2012;43:1969.

18. Misnaza Castrillón S. Drepanocitosis en Colombia: análisis de la notificación como enfermedad huérfana o rara al sistema de vigilancia en salud pública, 2016 y 2017. Bogotá: Instituto Nacional de Salud.; 2018. 15-01-2018. Contract No.: 14.

19. Arai Y. G6PD Deficiency: A Possible Cardiovascular Risk Factor in Older People. Journal of Atherosclerosis and Thrombosis. 2021;28(6):586-7.

20. República Sdl. Ley 1980 de 2019. POR MEDIO DE LA CUAL SE CREA EL PROGRAMA DE TAMIZAJE NEONATAL EN COLOMBIA. Bogotá: Congreso de Colombia; 2019. p. 1-7. 